\title{
Novel instruments for site characterization
}

\author{
J. W. V. Storey, M. C. B. Ashley and M. G. Burton \\ School of Physics, University of New South Wales, Sydney 2052, Australia
}

\begin{abstract}
In order to fully characterize the astronomical potential of remote sites on the antarctic plateau, we have developed a suite of instruments covering UV to sub-millimeter wavelengths. In addition, we have successfully demonstrated the use of an acoustic radar (SODAR) at the South Pole to measure the height of the turbulent atmospheric boundary layer. Each instrument is designed to operate independently and autonomously, producing reliable, fully calibrated data without human intervention. Although designed primarily for use in Antarctica, these instruments use novel technology that is applicable to other astronomical applications as well.
\end{abstract}

Keywords: Site-testing, astronomy, Antarctica

\section{INTRODUCTION}

\subsection{The importance of Antarctica for astronomy}

The antarctic plateau offers the promise of the best conditions on the surface of the Earth for a wide range of astronomical observations; see for example Burton et al $1994 .{ }^{1}$ Despite popular images of Antarctica as a harsh, windswept continent, conditions on the plateau are relatively benign. Cloud cover and precipitation are minimal and wind speeds average a low 3 meters per second. The main challenges are those of the altitude, isolation, and of course the extreme cold.

There are three properties of the antarctic environment that make it an exceptional site for astronomy. First, the atmospheric water vapor content at the South Pole in summer averages 0.2 to $0.5 \mathrm{~mm}$ of precipitable water vapor (PWV), falling below $0.1 \mathrm{~mm}$ PWV in winter. This dramatically improves the atmospheric transmission in the far-infrared and sub-millimeter windows over any other observing site on the surface of the Earth. ${ }^{2,3}$

Secondly, the antarctic plateau may also provide substantially better 'seeing' at optical wavelengths than even the best mountain top sites currently operating. ${ }^{4}$ The high altitude (over $4,000 \mathrm{~m}$ ), extreme cold (creating an equivalent pressure altitude of over $5,000 \mathrm{~m}$ ), small diurnal temperature changes and the absence of a high-altitude jet stream combine to minimize the microthermal fluctuations in the air temperature that degrade the spatial resolution of a telescope. In addition, the high altitude and reduced aerosol concentration in the atmosphere may lead to significantly enhanced UV transmission.

Thirdly, the thermal background radiation from the atmosphere and telescope is considerably reduced, leading to increased sensitivities. For example, in the near-infrared the thermal background is reduced by between one and two orders of magnitude from Mauna Kea at $0^{\circ} \mathrm{C}$ to the South Pole at $-60^{\circ} \mathrm{C}^{5-7}$ A particularly advantageous region is from 2.27 to $2.45 \mu \mathrm{m}$, where no airglow emission has been measured; the sky brightness here is up to a factor of 100 lower than at typical temperate observatories. This translates directly into a signal to noise gain of between 10 (for background-limited performance) and 100 (if sensitivity is limited by the ability to flat-field an array detector). Thus for near-IR observations, a telescope of 2 to 3 meter aperture could outperform the largest telescopes being built (with diameters of 10 to 16 meters) anywhere else on the Earth. At mid-infrared wavelengths the sky is also extremely dark, with flux levels falling to $5 \%$ of their typical 'temperate site' values. ${ }^{8,9}$

With the exception of some summer-time millimeter-wave observations at Dome $\mathrm{C}^{10}$ all of the site-testing measurements made to date have been carried out at the South Pole. This is because the Pole is currently the only station on the antarctic plateau with year-round operation.

It is very likely that sites higher on the plateau will have significantly better characteristics than the South Pole, which is at an elevation of only $2900 \mathrm{~m}$. However, it is difficult to justify developing other sites for which little or no site-testing data are available. In order to move forward we have developed a suite of instrumentation that

E-mail: j.storey@unsw.edu.au,m.ashley@unsw.edu.au,m.burton@unsw.edu.au 
can operate autonomously once installed at an isolated location. The instruments are designed for very low power operation and are powered from a thermoelectric generator fuelled by liquid propane.

This instrumentation, and the portable laboratory in which it is installed, can be deployed to a succession of potential antarctic plateau sites. It is intended that data will be collected at each of these sites for at least a year. The data will consist of:

- Atmospheric transmission from the UV cut-on to $800 \mathrm{~nm}$,

- Sky brightness at $2.4 \mathrm{~nm}$ resolution across the near-UV and visible,

- Sky brightness at $2.35 \mu \mathrm{m}$,

- Sky brightness at $2 \%$ spectral resolution from 4 to $14 \mu \mathrm{m}$,

- Optical depth and effective temperature of the atmosphere at $350 \mu \mathrm{m}$,

- Vertical distribution, with 20 meter resolution, of microthermal turbulence and three-dimensional wind velocity up to a height of 600 meters,

- Meteorological data such as temperature and atmospheric pressure.

These results will allow a quantitative assessment to be made of the relative advantages of each site. Combined with other factors such as accessibility and the availability of logistic support, the data will enable an informed decision to be made on the site for a major international observatory on the plateau.

\subsection{Existing antarctic observatories}

The potential of the antarctic plateau as a site for astronomy has been recognized for a number of years, and there are now several projects underway to explore this. The most developed come from the US group, CARA, which is currently operating three telescopes at the South Pole. These are a cosmic background experiment (VIPER), a sub-millimetre telescope (AST/RO) and most recently a cosmic microwave background interferometer (DASI). The SPIREX/Abu experiment, ${ }^{11}$ a $60 \mathrm{~cm}$ telescope with near-infrared array camera, has recently been decommissioned after acquiring two years' worth of excellent data-including, for example, the first wide-field thermal infrared images of the star-forming region NGC6334. ${ }^{12}$ There are also experiments at the Pole to measure cosmic rays, gamma rays and neutrinos, funded by individual grants from the National Science Foundation within the USA, and involving collaborators from Sweden, Germany and the UK.

Independent of these projects is the testing of millimeter and infrared telescopes by Italy on the antarctic coast (Terra Nova Bay), and preliminary measurements of the summer-time conditions at Dome C. ${ }^{10}$ A cosmic ray observatory is also operated by the Australian Antarctic Division at Mawson station on the coast.

\section{THE AUTOMATED ASTROPHYSICAL SITE TESTING OBSERVATORY (AASTO)}

Beginning in 1987, the NSF funded a program with Lockheed to develop a self-powered, self-heated field station to enable geophysical data to be obtained from the remote antarctic interior. This station, of which six were built, is called an Automated Geophysical Observatory. However, the basic station is equally suited to powering astronomical instruments and hence we have dubbed the seventh 'AGO', which has been built to our specifications, the Automated Astrophysical Site Testing Observatory, or AASTO. ${ }^{13,14}$

The AASTO is a very well insulated, small portable laboratory. Short-term accommodation is provided for up to four people, who accompany the AASTO to its destination, set up the experiments over a period of about one week, then leave the AASTO to operate autonomously for the next 12 months. A major challenge that Lockheed had to face was how to provide heat and electrical power for the full twelve months that the AASTO would sit on the ice in sub-zero temperatures. There is little wind on the plateau, it is dark for six months of the year, and environmental and cost considerations rule out radioisotope generators. The solution chosen is a propane-fuelled catalytic oxidizer that produces $2.5 \mathrm{~kW}$ of heat, plus 50 watts of electrical power via a thermoelectric generator.

The AASTO is designed to fit exactly into the cargo hold of a ski-equipped LC-130 ('Hercules') transport plane. In the initial 'put-in' flight, the AASTO will be placed on the snow, together with the science team of three or four persons and enough fuel for three weeks. Approximately one week later the Hercules will return with a year's supply of fuel and retrieve the science team. The AASTO can then remain on the ice for the next twelve months, gathering data in an autonomous mode very similar to that of a deep-space spacecraft. 


\subsection{Power management}

The AASTO thermoelectric generator produces a nominal 50 watts at 28 volts; this power bus is available for housekeeping power and for the instrumentation. Each instrument uses an independent, galvanically isolated DCDC converter to charge a 24 volt sealed lead-acid battery. Each battery charger is set so that it cannot draw more than 7 watts from the bus. As the instrument battery approaches a fully charged state, the battery charger reduces its drain on the bus to negligible values.

For most of the time the instruments, including their control computers, are powered down. The only activity is that of each instrument's 'wakey-wakey board', which counts $1 \mathrm{~Hz}$ clock pulses generated by a common clock in the AASTO housekeeping system. The wakey-wakey boards draw just 40 microamps each from the bus. Every two hours the board switches on power to the control computer of the instrument to which it is attached. The computer then consults an internal clock and other information, and decides whether or not to initiate a full power-up sequence for the instrument, or simply to allow itself to be shut down again. If it decides to switch the instrument on and take data, it then does so for a pre-determined time before allowing itself to be switched off. To remain on the computer must toggle a line to the wakey-wakey board every few seconds, otherwise it will automatically be shut down after 60 seconds. In this way a 'watchdog' function is implemented, automatically resetting the computer in the event of a software crash.

The computer monitors the battery voltage and can tell when the state of charge is starting to fall. It can then adjust the duty cycle of the instrument to ensure that the instrument is taking data for as much time as possible consistent with not exceeding its share of the available power.

\subsection{Instrument control and data management}

Each instrument is controlled by its own, independent computer running under the real-time multitasking operating system RTKernel. Each computer is built around a set of cards conforming to the PC/104 standard and typically uses a 486 processor plus flash memory. The observing software is written within a scriptable language called ERIC. ${ }^{15}$ Although operating independently, each computer also communicates via the RS-232 protocol with a central 'supervisor' computer. While the AASTO is still at the South Pole we have regular Internet access available. Communication from the University of NSW is made first to the supervisor computer, then through it to the individual instruments.

\subsection{Webcamera surveillance}

For the past two years we have monitored the AASTO with an Axis 200 webcamera. This has proved to be a most valuable resource, allowing us to check snow build up, sky conditions, and the physical status of the instruments. In January 2000 we added another three cameras: a tower-mounted camera with pan/tilt/zoom, a near-infrared camera with IR floodlamp for night-time monitoring of the G-mount, and a camera internal to the AASTO to monitor the electronics.

\subsection{Thermoelectric generator problems}

While the thermoelectric generator (TEG) and the thermosyphon that regulates the room temperature of the AASTO should be amongst the most reliable components of the system, we have experienced a succession of catastrophic failures. The particular failure mode is one in which the thermosyphon springs a leak, allowing Freon to enter the AASTO building. Because the TEG breathes room air, the Freon is drawn into the reaction chambers where it finds itself being passed over the red-hot platinum beads of the catalytic oxidizer. This decomposes the Freon into $\mathrm{HCl}$ and HF. The hot, gaseous acids then eat their way through the stainless-steel exhaust pipe and escape into the room. Once there, they cause devastating corrosion to the electronics, computers, and exposed parts of the instruments.

This particular failure mode is not unique to the AASTO, having been experienced in at least two of the other AGOs. It is clearly not a fundamental problem, and work is underway to implement some detailed design changes to prevent a recurrence. 


\section{THE AASTO INSTRUMENTS}

The AASTO instruments must be designed to operate under some unusual constraints:

- Autonomous operation,

- Total power budget of 50 watts (ie, 7 watts per instrument),

- Self calibrating,

- No maintenance or cryogen refills.

The key design philosophy has been one of imposing utmost simplicity on every system and subsystem. The AASTO environment allows room temperature operation of almost all components, dramatically simplifying the selection of parts and systems. However, some components must operate at temperatures as low as $-85^{\circ} \mathrm{C}$. In some cases, parts can be purchased off-the-shelf that already meet the low temperature specification. Nevertheless, it is always prudent to make exhaustive tests of critical components and, in doing so, it is sometimes found that inexpensive garden-variety components perform as well as their more highly specified cousins. To facilitate low temperature testing, we have installed in our laboratory a large Forma Scientific chest freezer of the type commonly used for storing biological specimens. Where instruments are too large to fit in the freezer, we find that a plywood box filled with dry ice makes a suitable test chamber.

\subsection{Antarctic Fiber-optic Spectrometer-AFOS}

The $\mathrm{AFOS}^{16}$ consists of a small telescope feeding a grating spectrometer and CCD detector via a bundle of six optical fibers. By observing bright stars, a direct measure of atmospheric transmission from the UV cut-on to about $800 \mathrm{~nm}$ is obtained. In addition, sky emission arising from aurorae and airglow can be monitored.

The AFOS telescope design is an $\mathrm{f} / 3.5$ Newtonian. Athermalization is achieved by using an Astrosittal mirror (a Russian-made ceramic similar to Zerodur) and an Invar telescope tube. The telescope tube is fully sealed (although provision is made for pressure equalization through a bi-directional blow-off valve), and has a $30 \mathrm{~cm}$ diameter optically flat fused-silica entrance window. A canister of $\mathrm{CaH}_{2}$ ensures that the partial pressure of water vapor inside the telescope remains below that which would condense out as ice, even at $-80^{\circ} \mathrm{C}$.

The AFOS uses a low-power telescope mount developed by the Australian National University (ANU) for their ADIMM (§3.6). This mount is intended to be a 'generic' mount for the AASTO project, and has thus become known as the 'G-mount'. The whole assembly is placed on a 7.5 meter high self-erecting tower (the 'G-tower') installed about 40 meters upwind from the AASTO itself. The idea is to place the telescopes above the worst of the surface-blown snow, and to keep them well away from the exhaust from the AASTO thermoelectric generator.

Two sets of three fibers bring light from the telescope to the AASTO. Each set has a central fiber with a 20 arcsecond field of view upon which the star will be focussed, and two 'sky' fibers to collect light for comparison spectra. One set of fibers is made from UV-enhanced material; the second set from low-OH silica with good red transmission. Light from the star is split with a dichroic beamsplitter and appropriately directed to the two fiber bundles. The beamsplitter, together with the poor blue transmission of the 'red' fibers, also acts as an order-sorting filter for the grating.

The spectrometer is a Jobin Yvon CP200 fixed-grating imaging spectrometer with extremely good scattered light performance. The individual spectra from all six fibers are simultaneously dispersed onto a $256 \times 1024$ pixel virtual phase, thermoelectrically cooled CCD. The CCD head and controller are manufactured by Andor Technology. The spectral resolution is approximately $2.4 \mathrm{~nm}$.

\subsection{Near-infrared sky monitor-NISM}

The NISM ${ }^{17}$ measures the near-infrared sky brightness, using an InSb detector and fixed $2.35 \mu \mathrm{m}$ filter cooled to $77 \mathrm{~K}$ by a low-power Stirling engine. A gold-coated reflective chopper wheel chops the received signal at a frequency of $77 \mathrm{~Hz}$ between two beams $45^{\circ}$ apart in elevation. Making a differential measurement in this way greatly improves the precision and removes many calibration uncertainties. The entire instrument is hermetically sealed, and looks out through a sapphire window. The optical system is arranged so that the two beams pass through exactly the same portion of the window, ensuring that any ice crystals that may have accumulated will affect both beams equally. 
A stepper motor drives the instrument about its elevation axis, allowing 'sky-dip' measurements to be carried out. Placed to one side of the instrument is a black-body calibration source, heated by the warmth of the AASTO building via a short length of OFHC copper rod.

The chopper is driven by a 3-phase brushless sensor-less motor, as only this type of motor will operate reliably and efficiently at the very low temperatures. A phase-locked loop is used to maintain a precise chopping frequency. The chopper motor, phase-locked loop and drive electronics together draw about 0.5 watts.

\subsection{Mid-infrared sky monitor-MISM}

Mid-infrared $(7-30 \mu \mathrm{m})$ astronomy also stands to gain from an antarctic plateau observatory. ${ }^{8}$ Although the drop in thermal background because of the lower temperature was predicted to be smaller than in the near-infrared, we have found the improvement to be much greater than expected. ${ }^{9}$ This results from a decrease in the emissivity of the sky to levels as low as $1 \%$. The MISM ${ }^{17}$ is a sky-brightness monitor similar in concept to its near-IR counterpart, but uses a Stirling-cycle cooled $\mathrm{HgCdTe}$ detector. A chopping frequency of $1 \mathrm{kHz}$ is used to avoid $1 / \mathrm{f}$ noise. An ambienttemperature filter wheel, driven by a stepper motor, carries a set of ambient temperature filters, giving spectral coverage from 4 to $14 \mu \mathrm{m}$. To reduce the background flux and improve the sensitivity, the instrument temperature is allowed to drop to $-30^{\circ} \mathrm{C}$. This temperature is maintained by a methanol-filled self-regulating heatpipe which transports warmth from the AASTO shelter.

During 1998 we collected a full 12 months of data with the MISM. The results show ${ }^{9}$ that the typical sky brightness on 'clear' days is just 20 Janskys/square arcsecond at $9.0 \mu \mathrm{m}$, and that the sky is clear for $50 \%$ of the time.

\subsection{Sub-mm tipper-SUMMIT}

This instrument measures the atmospheric transmission and effective atmospheric temperature at a wavelength of $350 \mu \mathrm{m}$. Originally constructed by the US National Radio Astronomy Observatory (NRAO) and Carnegie Mellon University, ${ }^{18}$ the sub-mm tipper uses a fixed-bandpass metal-mesh filter and a room-temperature pyroelectric detector. A stepper motor drives an off-axis paraboloid mirror, allowing the instrument to scan from zenith to horizon. Identical versions have been deployed to Mauna Kea (Hawaii), Chajnantor (Chile) and the South Pole. We are making substantial modifications to the unit we have acquired to improve its sensitivity and reduce the power consumption from 40 to 2.4 watts.

\subsection{Acoustic wind profiler-SODAR}

Measurements of the seeing at the South Pole have revealed typical values of around 1.7 arcseconds. ${ }^{19}$ However, microthermal measurements from a tower ${ }^{20}$ and from balloons ${ }^{21}$ have demonstrated that the bulk of the seeing disturbance on the antarctic plateau arises from the turbulent boundary layer, with a height generally less than $300 \mathrm{~m}$. This is in contrast to the situation at temperate observatory sites, where significant contributions arise from several layers distributed throughout the atmosphere-all the way from ground level to above the tropopause. Within the polar vortex there is no jet-stream, and the majority of the atmosphere appears to behave as a single, orderly layer. Measurement of the turbulence within the boundary layer is therefore expected to correlate extremely well with seeing.

It is also important to measure the height of the boundary layer. If this turbulent layer is sufficiently thin, it may be possible to place a future large telescope completely above it simply by constructing a modestly sized tower. At Dome $\mathrm{C}$, and other high plateau sites, the wind speed is much lower than at the Pole. This in turn should lead to a much thinner boundary layer, possibly as low as a few meters in some circumstances.

Since balloon measurements are impractical from an uninhabited location, we explored all possible alternative methods of characterizing the lower atmosphere. We concluded that the task can best be done by an acoustic wind profiler, or 'SODAR'. A SODAR is the acoustic analog of a radar and measures microthermal turbulence directly, as the intensity of the echoes are directly proportional to $\mathrm{C}_{\mathrm{T}}^{2}$. Range gating is used to determine the distance to the elements of air responsible for each reflection, while Doppler analysis allows wind velocities to be calculated. By employing three acoustic beams (one vertical, two slanted at $45^{\circ}$ ), the three-dimensional wind profile can also be obtained.

This unit we have purchased is a model PA-1, manufactured by Remtech (France). It is capable of a height resolution of 20 meters, and has a range of up to 800 meters under normal circumstances. It is very compact, and has a fixed, low-profile acoustic antenna. The basic principal is similar to a phased-array radar, the antenna 
consisting of an electrically steerable array of 52 piezoelectric drivers. The PA1 is designed for battery powered use, and requires only 25 watts. This can be readily accommodated within our nominal AASTO power budget of 7 watts per instrument by running at a $25 \%$ duty cycle throughout the year (eg, 1 hour on, three hours off).

We adapted the SODAR for antarctic use by stretching a light, nylon cover across the top of the antenna. By vibrating like a drum head in light winds, the cover is extremely effective at preventing the build up of snow on the antenna. The cover appears to have negligible acoustic attenuation and hence little effect on the range. We discarded the electrical heating tape that is normally used to warm the antenna in cold climates, as we do not have electrical power to spare for such purposes. Instead, the antenna was placed over the top of one of the 16-inch diameter open ports in the roof of the AASTO. This allows warm AASTO room air to heat the antenna from below. Tests in our simulation facility have previously shown that the piezo-electric drivers used in the Remtech SODAR continue to work all the way down to $-85^{\circ} \mathrm{C}$, but with some loss of efficiency.

We installed the SODAR on top of the AASTO in January 1999. Its performance was found to be more than adequate for our needs, consistently returning echoes from up to $600 \mathrm{~m}$ altitude despite the low values of $\mathrm{C}_{\mathrm{T}}^{2}$ expected at the South Pole.

\subsection{ADIMM}

In addition to the instruments described above, the Australian National University (ANU) is developing an instrument known as the ADIMM (Antarctic Differential Image-Motion Monitor). ${ }^{22}$ This will directly measure the seeing by monitoring the relative motion of a set of stellar images at the focal plane of a $35 \mathrm{~cm}$ telescope. Each image is generated by a separate sub-aperture in the pupil plane of the telescope. The ADIMM was deployed to the South Pole on one side of the ANU's G-mount in January 2000.

\section{THE FUTURE}

The AASTO was officially opened at the South Pole on January 9, 1997, by Senator Robert Hill, the Australian Minister for the Environment. For the past three years it has gathered a significant amount of useful data, despite a series of catastrophic failures of the thermoelectric generator. Over the next four years we plan to pursue the following timetable:

2000: The NISM and SODAR have been installed on the AASTO and are currently returning data. The thermoelectric generator has been removed and returned to the US for complete rebuilding-all instruments are running on station power for this year only. The MISM and SUMMIT are currently at UNSW undergoing further development. The AFOS is installed on the G-mount in a mode in which it can track objects in azimuth only. During 2000 we expect to acquire a full data set from the NISM and SODAR, and to obtain useful data from the AFOS as the moon passes through the fixed-elevation telescope beam.

2001: The AASTO will operate for the first time with its full complement of site-testing instruments, powered entirely by the rehabilitated thermoelectric generator. A year of testing at the South Pole will properly qualify the system for deployment to a remote site, while nevertheless giving us the opportunity to correct any unforeseen problems.

2002: We plan to move the AASTO and its instruments to the French-Italian station at Dome C, carrying out the first comprehensive, year-long assessment of the potential of that site.

2003: The next focus for the AASTO and its instruments will be sites still higher on the plateau. Perhaps the most viable of these is the Russian station at Vostok. Ultimately, we hope to be able to deploy the AASTO to the summit of Dome A, the highest point on the antarctic plateau.

\section{ACKNOWLEDGEMENTS}

The AASTO project is a collaboration between the University of NSW, the Australian National University and the US Center for Astrophysical Research in Antarctica. The UNSW work is supported by the Australian Research Council. We particularly thank Jack Doolittle for his valuable advice, and Max Boccas, Andre Phillips, Ant Schinckel, Jon Everett and Paolo Calisse for their many contributions to the instrumentation. Special thanks are reserved for Bob Pernic and Robert Spotz, whose skills at the South Pole have proved invaluable. 


\section{REFERENCES}

[1] M.G. Burton et al, "The scientific potential for astronomy from the antarctic plateau", Publ. Astron. Soc. Aust., 11, pp. 127-150, 1994.

[2] R.A. Chamberlin, A.P. Lane and A.A. Stark, "The $492 \mathrm{GHz}$ opacity at the Geographic South Pole", Astrophys. J., 476, pp. 428-433, 1997.

[3] A.P. Lane, "Submillimeter transmission at South Pole", in Astrophysics from Antarctica, G. Novak and R.H. Landsberg, eds., ASP Conference Series, 141, pp. 289-295, 1998.

[4] P.R. Gillingham, "Super seeing from the Australian Antarctic Territory?", ANARE Research Notes, 88, pp. 290-292, 1992.

[5] M.C.B. Ashley, M.G. Burton, J.W.V. Storey, J.P. Lloyd, J.W. Briggs and D.A. Harper, "South Pole observations of the near-infrared sky brightness," Publ. Astron. Soc. Pac., 108, pp. 721-723, 1996.

[6] H.T. Nguyen, B.J. Rauscher, S.A. Severson, M. Hereld, D.A. Harper, R.F. Loewenstein, F. Mrozek, and R.J. Pernic, "The South Pole near-infrared sky brightness", Publ. Astron Soc. Pac. 108, pp. 718-720, 1996.

[7] M.A. Phillips, M.G. Burton, M.C.B. Ashley, J.W.V. Storey, J.P. Lloyd, D.A. Harper and J. Bally, "The nearinfrared sky emission at the South Pole in winter", Astrophys J., 527, pp. 1009-1022, 1999.

[8] C.H. Smith and D.A. Harper, "Mid-infrared sky brightness testing at the South Pole", Publ. Astron Soc. Pac. 110, pp. 747-753, 1998.

[9] M.A. Chamberlain, M.C.B. Ashley, M.G. Burton, A. Phillips, J.W.V. Storey and D.A. Harper, "Mid-Infrared Observing Conditions at the South Pole", Astrophys. J., in press, 2000.

[10] L. Valenziano and G. Dall'Oglio, "Millimetre astronomy from the high antarctic plateau: site testing at Dome C", Publ. Astron. Soc. Aust., 16, pp. 167-174, 1999.

[11] A.M. Fowler, N. Sharp, W. Ball, A.E.T. Schinckel, M.C.B. Ashley, M. Boccas, J.W.V. Storey, D. Depoy, P. Martini, D.A. Harper, and R.D. Marks, "ABU/SPIREX: The South Pole thermal IR experiment", Proc SPIE 3354, pp. 1170-1178, 1998.

[12] M.G. Burton, M.C.B. Ashley, R.D. Marks, A.E.T. Schinckel, J.W.V. Storey, A. Fowler, M. Merrill, N. Sharp, I. Gatley, D.A. Harper, R.F. Loewenstein, F. Mrozek, J. Jackson, K. Kraemer, "High resolution imaging of photodissociation regions in NGC 6334", Astrophys. J., submitted, 2000.

[13] J.W.V. Storey, M.C.B. Ashley and M.G. Burton, "An Automated Astrophysical Observatory for Antarctica", Publ. Astron. Soc. Aust., 13, pp. 35-38, 1996.

[14] J.W.V. Storey, "The AASTO Program", in Astrophysics from Antarctica, G. Novak and R.H. Landsberg, eds., ASP Conference Series, 141, pp 313-318, 1998.

[15] M.C.B. Ashley, P.W. Brooks and J.P. Lloyd, "Remote control of astronomical instruments via the Internet", Publ. Astron. Soc. Aust., 13, pp. 17-21, 1996.

[16] M. Boccas, M.C.B. Ashley, M.A. Phillips, A.E.T. Schinckel and J.W.V. Storey, "Antarctic Fiber Optic Spectrometer", Publ. Astron. Soc. Pac., 110, pp. 306-316, 1998.

[17] J.W.V. Storey, M.C.B. Ashley, M. Boccas, M.A. Phillips and A.E.T Schinckel, "Infrared sky brightness monitors for Antarctica", Publ. Astron. Soc. Pac., 111, pp. 765-771, 1999.

[18] P.A.R. Ade, R.A. Chamberlin, M.J. O'Kelly, J.B. Peterson, S.J.E. Radford and E. Schartman, "Comparison of the sub-millimeter opacity and sky stability at Mauna Kea, Chajnantor and South Pole", in preparation, 2000.

[19] R.F. Loewenstein, C. Bero, J.P. Lloyd, F. Mrozek, J., Bally, and D. Theil, "Astronomical seeing at the South Pole", in Astrophysics from Antarctica, G. Novak and R.H. Landsberg, eds., ASP Conference Series, 141, pp. 296-302, 1998.

[20] R.D. Marks, J. Vernin, M. Azouit, J.W. Briggs, M.G. Burton, M.C.B. Ashley, and J.F. Manigault, "Antarctic site testing-microthermal measurements of surface-layer seeing at the South Pole", Astron. \& Astrophys. Suppl. 118, pp. 385-390, 1996.

[21] R.D. Marks, J. Vernin, M. Azouit, J.F. Manigault and C. Clevelin, "Measurement of optical seeing on the high antarctic plateau", Astron. \& Astrophys. Suppl. 134, pp. 161-172, 1999.

[22] M.A. Dopita, P.R. Wood and G.R. Hovey, "An automated DIMM telescope for Antarctica", Publ. Astron. Soc. Aust., 13, pp. 39-43, 1996. 